\title{
Virtual Noon Conferences: Providing Resident Education and Wellness During the COVID-19 Pandemic
}

\author{
Thomas W. Hahn, MD
}

PRIMER. 2020;4:17.

Published: 8/14/2020 | DOI: 10.22454/PRiMER.2020.364166

\section{Abstract}

Introduction: The COVID-19 pandemic has drastically impacted graduate medical education. Family medicine residents are now doing substantial clinical work and learning from home. We continued to offer academic half-day didactics virtually, but sensed a need for daily resident education and social support, so we implemented a virtual daily noon conference to address these needs.

Methods: The virtual noon conferences used web-based technology and had weekly organ system themes with consistent daily learning activities like cases and review questions. Four key components made the conferences collaborative and inclusive; they were led by residents, required minimal preparation by using available materials, were interactive, and promoted social connection with wellness activities. We evaluated the impact on resident-perceived knowledge and wellness over 6 weeks with weekly surveys for residents attending at least one conference that week and a postintervention survey.

Results: Of 66 responses to the weekly surveys, $98 \%$ agreed that noon conferences helped to increase knowledge and social connection. Of 46 total residents, 35 (76\%) answered the postintervention survey, and all agreed that noon conferences helped to increase knowledge, were interactive, and increased social connection. Ninety-one percent favored continuing the noon conferences. More than three-quarters of postintervention survey respondents agreed that virtual noon conferences were equally or more beneficial compared to academic half days for both their education and well-being during the pandemic.

Conclusions: Virtual noon conferences have provided regular interactive learning and fostered resident wellbeing during the pandemic. They can supplement resident curriculum and wellness and can be easily adopted by other programs.

\section{Introduction}

The COVID-19 pandemic has drastically impacted health care and graduate medical education. Many nonessential clinical services and learning opportunities have transitioned to electronic formats. Family medicine residents are doing a significant amount of clinical work and independent learning from home when not working in the hospital. During this time of physical distancing, there is a need to adapt educational opportunities and provide support.

Traditionally, residency programs have offered didactic learning via daily noon conferences; however, over the past decade, many residency programs like ours have shifted to weekly academic half days (AHD), which involve a 3- to 4-hour didactic block once weekly. AHD allow for protected learning time, active learning, and deliberate curriculum 
structuring. ${ }^{1}$ Compared to noon conferences, AHD may improve resident attendance and medical knowledge, reduce interruptions, and increase resident and faculty satisfaction. ${ }^{1-4}$

When the pandemic started, we continued to offer AHD virtually. When residents in our program were not working in the hospital, they were doing the majority of their learning and continuity clinical care from home and expressed a desire for daily structured learning. We also wanted to provide an opportunity for regular social connection during this unprecedented time.Thus, we implemented a virtual daily noon conference and evaluated its impact on resident learning and well-being compared to AHD during the pandemic.

\section{Methods}

Residents from a university-based family medicine residency program participated in the study. We invited all 46 residents to participate and evaluate the intervention. This study was exempt from review by our university institutional review board.

\section{Curriculum}

We implemented a 1-hour weekday virtual noon conference using web-based video and teleconference technology. Each week was organized by organ system (cardiology, pulmonology, etc), covered the breadth of family medicine (outpatient, inpatient, adult, pediatrics), and had consistent daily learning activities (Monday adult topic review, Tuesday case discussion, Wednesday pediatric topic review, Thursday guidelines/evidence review, Friday board review questions). There was a presentation on a COVID-19 topic every 2 weeks.

To promote a collaborative, inclusive learning environment, we built the conferences around four key components; they were led by residents, required minimal preparation, were interactive, and promoted wellness and social connection (Figure 1). Conferences were open to all residents and residency faculty. One or more residents led the conference each day and chose the daily topic. A faculty coordinator helped to organize the conferences by selecting weekly themes and identifying resources. We used available materials from medical societies and our medical school library that required minimal preparation. The conferences used interactive approaches like case presentations, questions, and literature searches. To promote social connection, each conference started with a wellness activity like a mindfulness meditation or virtual game.

\section{Evaluation}

Residents evaluated the conference over 6 weeks with weekly surveys for those attending at least one conference that week and with a postintervention survey. Both surveys asked questions about impact on perceived knowledge, quality of learning activities, and impact on social connection using a 4-point Likert scale (1=strongly agree, 4=strongly disagree). The postintervention survey also asked about preparation time, desire to continue the noon conferences in the future, and the benefit of virtual noon conferences for learning and wellness compared to AHD during the pandemic.

\section{Results}

There were approximately 25 daily attendees at the virtual noon conference. Of 66 responses to the weekly surveys, $98 \%$ agreed that noon conferences helped to expand knowledge, contained useful learning activities, and improved social connection.

Thirty-five of 46 total residents (76\%) completed the postintervention survey. For 16 respondents who had led a conference, preparation time was less than 30 minutes for $31 \%, 30-60$ minutes for $31 \%$, and more than 60 minutes for $38 \%$. One hundred percent of respondents agreed that noon conferences helped to increase knowledge, were interactive, and helped them feel socially connected to other residents. Ninety-one percent of postintervention survey respondents favored continuing the noon conferences in the future. Compared to AHD during the pandemic, $80 \%$ of respondents agreed that noon conferences were equally or more beneficial for their learning, and $77 \%$ agreed 


\section{Conclusions}

\section{Summary of Findings}

Virtual noon conferences have been a welcome addition to our residency program during an uncertain time by providing consistent, resident-led, interactive education that requires minimal preparation for most residents and has improved resident-perceived knowledge and social connection. At least three-quarters of residents reported that noon conferences were equally or more beneficial compared to AHD for education and well-being during the pandemic.

\section{Significance of Findings}

There will be many lasting changes to health care and medical education as a result of the pandemic. While it is unlikely that noon conferences will replace AHD, they may be a beneficial addition to some programs as we enter a new normal. The virtual nature makes attendance at noontime conferences more realistic, and virtual noon conferences offer what AHD cannot: structured learning and social connection on a daily basis. The value of an education conference is not necessarily determined by the duration but rather by the type and quality of the learning activities, particularly interactive, case-based learning. ${ }^{5,6}$ Noon conferences may provide an opportunity to structure curriculum in new ways, such as organizing by organ system or developing resident-led conferences.

Noon conferences can also impact resident wellness. Social support is an important part of resident wellness, ${ }^{7,8}$ and while some studies show that transitioning from noon conferences to AHD can benefit resident wellness, ${ }^{2}$ we found that adding noon conferences increased social connection and well-being during a time of physical distancing.

\section{Study Limitations}

Our study sample size was small and from a single institution, so may not be generalizable. The weekly surveys did not track individual responses over time.

\section{Future Directions}

As we settle into a new normal, residents at our program want to continue virtual noon conferences in addition to AHD. We will continue to adjust the frequency and content of the noon conferences as our schedules evolve and will consider further evaluating the impact of the conferences on knowledge acquisition and wellness indicators.

\section{Conclusion}

Virtual noon conferences have been a simple yet effective solution for structured learning and social connection during the COVID-19 pandemic and potentially beyond, reminding us that sometimes the best solutions are found in the past and adapted to meet the needs of the present. 


\section{Tables and Figures}

Figure 1: Four Key Components of Collaborative Virtual Noon Conferences

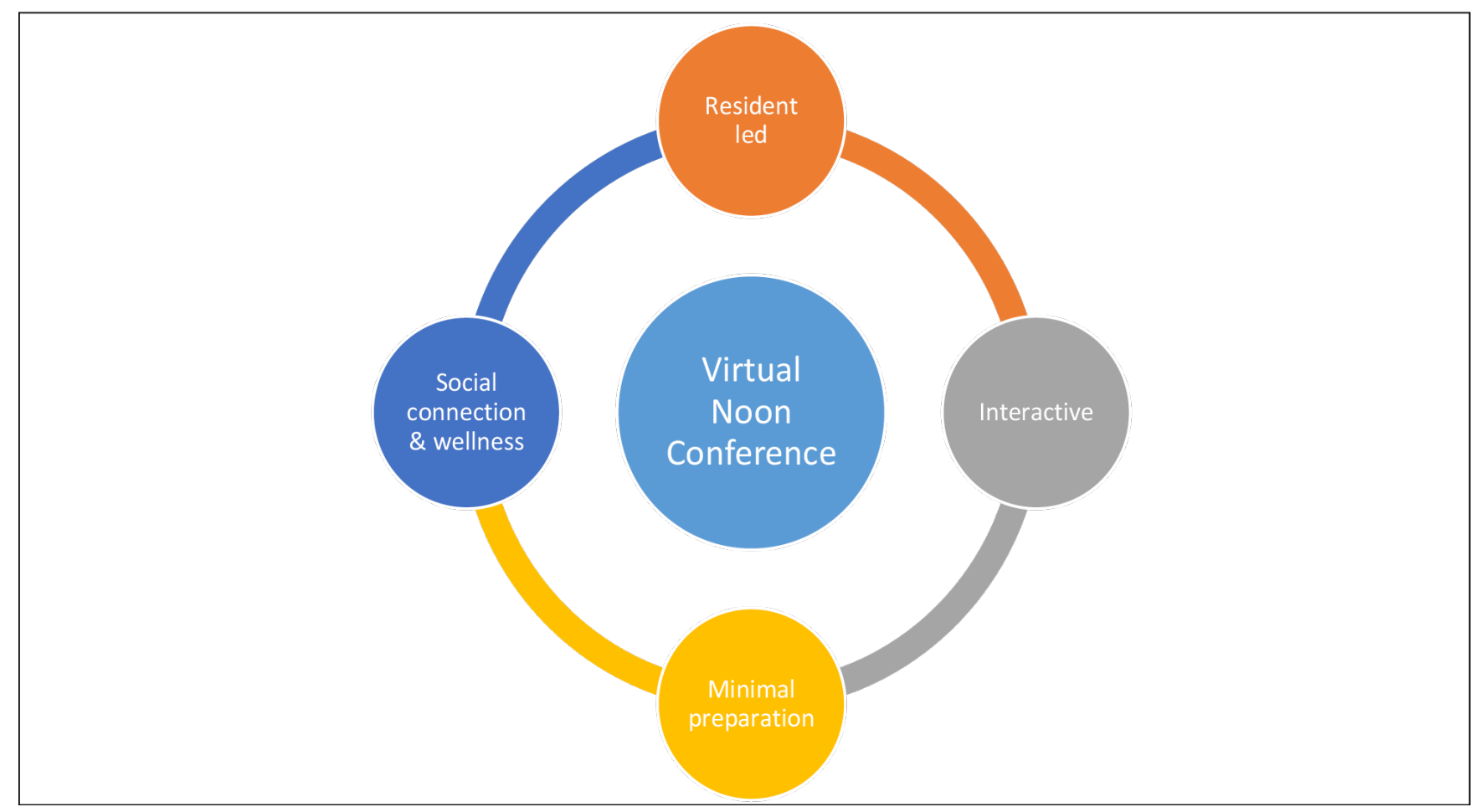




\section{Figure 2: Impact of Virtual Noon Conferences vs Academic}

Half Days on Learning and Well-being $(\mathrm{N}=35)$

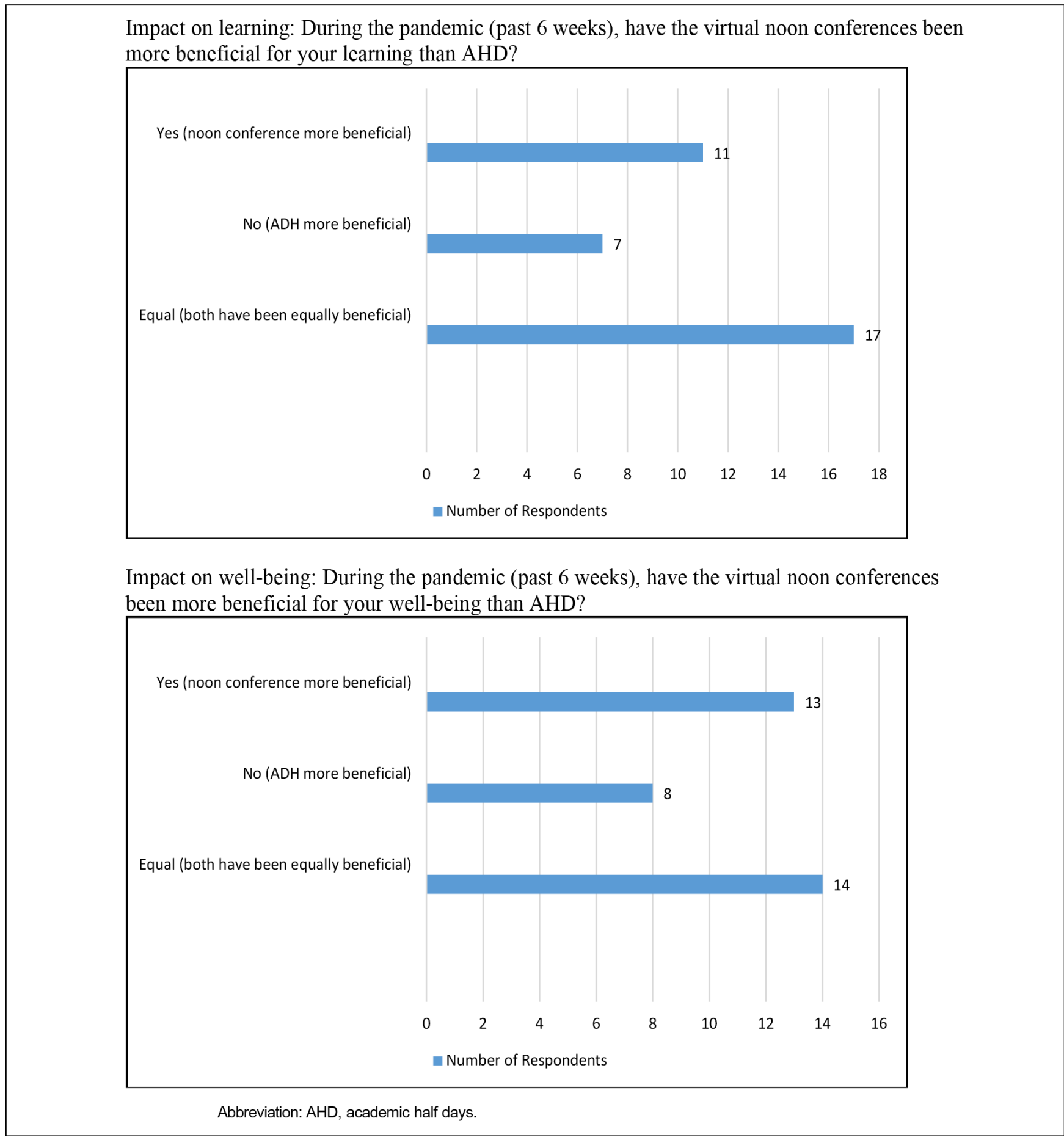

\section{Acknowledgments}

The author thanks John Frey, MD, for assistance with conceptual development and to Sarina Schrager, MD, MS, and Mindy Smith, MD, MS, for guidance with the manuscript.

\section{Corresponding Author}

Thomas W. Hahn, MD

100 N Nine Mound Rd, Verona, WI 53593.608-845-9531. Fax: 608-845-8684. thomas.hahn@fammed.wisc.edu. 


\section{Author Affiliations}

Thomas W. Hahn, MD - University of Wisconsin School of Medicine and Public Health, Department of Family Medicine and Community Health

\section{References}

1. Batalden MK, Warm EJ, Logio LS. Beyond a curricular design of convenience: replacing the noon conference with an academic half day in three internal medicine residency programs. Acad Med. 2013;88(5):644-651. doi:10.1097/ACM.0b013e31828b09f4

2. Zastoupil L, Mclntosh A, Sopfe J, et al. Positive impact of transition from noon conference to academic half day in a pediatric residency program. Acad Pediatr. 2017;17(4):436-442. doi:10.1016/j.acap.2017.01.009

3. Ha D, Faulx M, Isada C, et al. Transitioning from a noon conference to an academic half-day curriculum model: effect on medical knowledge acquisition and learning satisfaction. J Grad Med Educ. 2014;6(1):93-99. doi:10.4300/JGME-D-13-00185.1

4. Moreno MA, Kota R, McIntosh GC, Frohna JG. PEARLs of wisdom: impact of a new block conference on pediatrics resident attendance, satisfaction, and learning. J Grad Med Educ. 2013;5(2):323-326. doi:10.4300/JGME-D-12-00249.1

5. Sawatsky AP, Berlacher K, Granieri R. Using an ACTIVE teaching format versus a standard lecture format for increasing resident interaction and knowledge achievement during noon conference: a prospective, controlled study. BMC Med Educ. 2014;14(1):129. doi:10.1186/1472-6920-14-129

6. Sawatsky AP, Zickmund SL, Berlacher K, Lesky D, Granieri R. Understanding resident learning preferences within an internal medicine noon conference lecture series: a qualitative study. J Grad Med Educ. 2014;6(1):32-38. doi:10.4300/JGME-06-01-37.1

7. Raj KS. Well-being in residency: a systematic review. J Grad Med Educ. 2016;8(5):674-684. doi:10.4300/JGME-D-15-00764.1

8. Parsons M, Bailitz J, Chung AS, et al. Evidence-based interventions that promote resident wellness from the council of emergency residency directors. West J Emerg Med. 2020;21(2):412-422.

doi:10.5811/westjem.2019.11.42961

Copyright $\odot 2020$ by the Society of Teachers of Family Medicine 